\title{
Methylation and Expression of Immune and Inflammatory Genes in the Offspring of Bariatric Bypass Surgery Patients
}

\author{
Frédéric Guénard, ${ }^{1,2}$ André Tchernof, ${ }^{2,3}$ Yves Deshaies, ${ }^{3,4}$ Katherine Cianflone, ${ }^{3,4}$ \\ John G. Kral, ${ }^{5}$ Picard Marceau, ${ }^{3,6}$ and Marie-Claude Vohl ${ }^{1,2}$ \\ ${ }^{1}$ Department of Food Science and Nutrition, Institute of Nutrition and Functional Foods (INAF), Laval University, \\ Québec, QC, Canada G1V 0A6 \\ ${ }^{2}$ Endocrinology and Nephrology, CHU de Québec Research Center, Québec, QC, Canada G1V 4G2 \\ ${ }^{3}$ Québec Heart and Lung Institute, Québec, QC, Canada G1V 4 G5 \\ ${ }^{4}$ Department of Medicine, Laval University, Québec, QC, Canada G1V 0A6 \\ ${ }^{5}$ Department of Surgery, SUNY Downstate Medical Center, Brooklyn, NY 11203, USA \\ ${ }^{6}$ Department of Surgery, Laval University, Québec, QC, Canada G1V 0A6
}

Correspondence should be addressed to Marie-Claude Vohl; marie-claude.vohl@fsaa.ulaval.ca

Received 28 March 2013; Revised 17 May 2013; Accepted 22 May 2013

Academic Editor: Matthias Bluher

Copyright (C) 2013 Frédéric Guénard et al. This is an open access article distributed under the Creative Commons Attribution License, which permits unrestricted use, distribution, and reproduction in any medium, provided the original work is properly cited.

Background. Maternal obesity, excess weight gain and overnutrition during pregnancy increase risks of obesity, type 2 diabetes mellitus, and cardiovascular disease in the offspring. Maternal biliopancreatic diversion is an effective treatment for severe obesity and is beneficial for offspring born after maternal surgery (AMS). These offspring exhibit lower severe obesity prevalence and improved cardiometabolic risk factors including inflammatory marker compared to siblings born before maternal surgery (BMS). Objective. To assess relationships between maternal bariatric surgery and the methylation/expression of genes involved in the immune and inflammatory pathways. Methods. A differential gene methylation analysis was conducted in a sibling cohort of $25 \mathrm{BMS}$ and 25 AMS offspring from 20 mothers. Following differential gene expression analysis (23 BMS and 23 AMS), pathway analysis was conducted. Correlations between gene methylation/expression and circulating inflammatory markers were computed. Results. Five immune and inflammatory pathways with significant overrepresentation of both differential gene methylation and expression were identified. In the IL-8 pathway, gene methylation correlated with both gene expression and plasma C-reactive protein levels. Conclusion. These results suggest that improvements in cardiometabolic risk markers in AMS compared to BMS offspring may be mediated through differential methylation of genes involved in immune and inflammatory pathways.

\section{Introduction}

Parental obesity increases the risk of obesity in offspring through genetic, biological, and environmental influences [13]. Maternal obesity, weight gain, and increased body mass index (BMI) between pregnancies and gestational diabetes increase risks of offspring obesity, type 2 diabetes mellitus (T2DM), and cardiovascular disease (CVD) in the offspring [4-7]. Consistent with the chronic low-grade inflammatory state of obesity, elevated concentrations of interleukin-6 (IL6) and C-reactive protein (CRP) have been observed in obese pregnant women [8]. Higher maternal levels of IL-6 were predictive of the increased growth and adiposity in the offspring [9]. Maternal diet-induced obesity in sheep resulted in higher fetal triglyceride (TG) levels [10] and upregulated inflammatory signaling [11]. Offspring of rats injected with IL-6 throughout pregnancy had an altered inflammatory profile associated with greater body fat mass and reduced insulin sensitivity [12].

Epigenetic processes are important mediators of earlylife environment, metabolism, and body composition in offspring. Epigenetic changes in genes involved in the inflammatory response have been observed in response to maternal diet and adiposity. Differences in methylation levels of the 
peroxisome proliferator-activated receptor alpha (PPARA) and glucocorticoid receptor promoters have been observed in the liver of offspring of rats fed with protein-restricted diet during pregnancy $[13,14]$. Methylation status of retinoid $\mathrm{X}$ receptor alpha (RXRA) promoter in umbilical cord tissue DNA was correlated with increased adiposity in children of mothers with lower carbohydrate intake [15]. Epigenetic mechanisms have been involved in inflammation and associated disorders including obesity $[15,16]$.

Bariatric surgery is known to improve glucose and lipid metabolism [17-19] and prevent arterial hypertension and type 2 diabetes [19-21]. Bariatric surgery also decreases the low-grade inflammation associated with obesity [22]. In previous studies, we demonstrated that children born after maternal bariatric surgery (AMS) exhibited lower prevalence of severe obesity, greater insulin sensitivity, and improved lipid profile in comparison to siblings born before maternal surgery (BMS) [23, 24]. In addition, AMS children demonstrated differences in a marker of chronic low-grade inflammation, namely the plasma C-reactive protein (CRP) [24].

This study was designed to analyze the relationship between maternal biliopancreatic diversion surgery and methylation levels of genes involved in immunologic and inflammatory pathways in BMS and AMS offspring. Pathway analyses were conducted using both methylation and expression data. Correlation analyses between gene methylation, expression, and plasma CRP levels were conducted for the IL8 pathway.

\section{Material and Methods}

2.1. Subjects. Women from Québec City and surrounding areas (administrative regions of Capitale-Nationale, Mauricie, and Chaudière-Appalaches) who had given birth before and after biliopancreatic diversion with duodenal switch for severe obesity were eligible for the current study. A subset of 20 unrelated mothers was recruited along with 50 siblings born before and after surgery (25 BMS and 25 AMS offspring). Mothers and offspring attended the Québec Heart and Lung Institute (Québec City, Québec, Canada) or a regional hospital for assessment between July and October 2010. There were 17 mothers with siblings born before and after surgery (23 BMS and 24 AMS), two with BMS offspring only (2 BMS), and one mother with only one AMS offspring. This study was approved by the Québec Heart and Lung Institute Ethics Committee. Written informed consent was obtained from adults and mothers with assents from minors.

Maternal presurgical data were obtained from medical records. Percent body fat was determined for individuals at 6 years or more (BMS, $N=23$; AMS, $N=17$ ) using bioelectric impedance analysis (Tanita, Arlington Heights, IL, USA). Weight, height, and resting systolic (SBP) and diastolic (DBP) blood pressure were obtained using standardized procedures and measured at the interview. BMI was calculated for mothers, and BMI percentile for children was obtained from the National Health and Nutrition Examination Survey 2000 charts [25]. BMI Z-score was calculated for children using charts from the Centers for Disease Control and Prevention [26]. Severe obesity in offspring was defined using ageand sex-corrected adiposity measurements (BMI $Z$-score > 3; BMI percentile > 98\%). Blood samples were collected from mothers and offspring after an overnight fast into BD Vacutainer tubes containing EDTA and PAXgene Blood RNA collection tubes (Qiagen, Valencia, CA, USA). Plasma TG, high-density lipoprotein cholesterol (HDL-C), low-density lipoprotein cholesterol (LDL-C), total cholesterol (total-C), total-C/HDL-C ratio, glucose, and insulin concentrations were measured as previously described [27]. The homeostatic model of insulin resistance (HOMA-IR) index was calculated as glucose $\times$ insulin/22.5. Levels of high-sensitivity CRP levels were measured by the clinical biochemistry laboratory. CRP values under the detection limit $(<0.17 \mathrm{mg} / \mathrm{L})$ were arbitrarily set at detection limit.

2.2. DNA Methylation Analysis. Genomic DNA was isolated from the blood buffy coat using the GenElute Blood Genomic DNA Kit (Sigma, St. Louis, MO, USA) and quantified using both NanoDrop Spectrophotometer (Thermo Scientific, Wilmington, DE, USA) and PicoGreen DNA methods. DNA $(1 \mu \mathrm{g})$ was bisulfite converted, and quantitative genomewide methylation analysis was conducted using the Infinium HumanMethylation450 BeadChip (Illumina, San Diego, CA, USA). Arrays were processed at the McGill University and Génome Québec Innovation Centre (Montréal, Canada) according to the manufacturer's instructions (Illumina, San Diego, CA, USA). The Infinium HumanMethylation450 BeadChip array was designed for genome-wide methylation analysis with coverage targeted across gene regions with sites in the promoter region, $5^{\prime} \mathrm{UTR}$, first exon, gene body, and $3^{\prime}$ UTR. The BeadChip interrogates more than 485000 methylation sites at single-nucleotide resolution.

Visualization and analysis of methylation data were conducted using the GenomeStudio software version 2011.1 (Illumina Inc.) and the Methylation Module. Methylation levels (beta values; $\beta$ ) were estimated as the ratio of signal intensity of the methylated alleles to the sum of methylated and unmethylated intensity signals of the alleles $(\beta$ value $=$ $\mathrm{C} /(\mathrm{T}+\mathrm{C}))$. Internal control probe pairs were used for data correction (background subtraction and normalization), and CpG sites with a detection $P$ value $>0.05$ were removed from analysis. Differences in methylation levels between BMS and AMS groups (mean $\beta$ values) were tested using Illumina Custom model. False-discovery-rate-corrected (FDRcorrected) $P$ values and DiffScores were computed. FDR cutoff of $\leq 5 \%$ was applied to correct for multiple testing. DiffScore is a differential methylation score calculated from $P$ values and differences in $\beta$ (delta $\beta$ ) between the two groups and was used here as the main statistical results for comparison of BMS and AMS siblings. Briefly, a call rate of $99.95 \%$ was obtained for methylation data. Differential methylation analysis between BMS and AMS siblings revealed 5698 genes (14 466 probes) with significant differences in methylation levels (FDR-corrected DiffScore $\geq|13| \sim$ $P \leq 0.05)$. Distribution of differentially methylated probes obtained from comparison between BMS and AMS offspring 
is provided in Supplementary Table 1 available online at http://dx.doi.org/10.1155/2013/492170.

2.3. Gene Expression Analysis. Total RNA was isolated and purified from whole blood for 46 offsprings (23 BMS and 23 AMS) using PAXgene Blood RNA Kit (Qiagen) following the manufacturer's recommendations. The integrity of the purified RNA was analyzed using both NanoDrop (Thermo Scientific, Wilmington, DE, USA) and 2100 Bioanalyzer (Agilent Technologies, Cedar Creek, TX, USA). Expression levels were measured using the HumanHT-12 v4 Expression BeadChip (Illumina Inc.) which contains more than 47000 probes derived from NCBI RefSeq Release 38. Microarray experiments were carried out using $250 \mathrm{ng}$ of total RNA and processed according to the manufacturer's instructions at the McGill University and Génome Québec Innovation Centre (Montréal, Canada). Expression data were visualized and analyzed using the FlexArray software (version 1.6) [28], and the Lumi algorithm was used for expression data analysis and normalization. Probe detection analysis was conducted using the FlexArray filter algorithm to generate a list of expressed probes. To be considered as significantly expressed, a probe had to show a detection $P$ value $\leq 0.05$ in at least $25 \%$ of the samples of a group. The "Significance Analysis of Microarrays" (SAM) algorithm [29] with unequal-variance (Welch's) $t$-statistic was used to assess differences between BMS and AMS siblings for significantly expressed probes. The SAM algorithm uses permutations of the data set to generate an empirical null distribution and to assess the significance of observed effects, rather than using the hypothetical $t$ distribution. A cutoff of $P \leq 0.05$ was used to detect differentially expressed probes. In addition, a cutoff of fold change $\geq 1.2$ or $\leq 0.83$ (symmetrical fold change $\geq 1.2$ or $\leq-1.2$ ) was used. $P$ values obtained from permutations and fold change cutoff values were then used to minimize the chances of false positives. Differential expression analysis revealed a total of 862 probes differentially expressed with a symmetrical fold change $\geq|1.2|(P \leq 0.05$; fold change $<0.83$ or $>1.2)$.

Gene expression microarray results were validated using real-time polymerase chain reaction (RT-PCR; Applied Biosystems Gene Expression Assays; Applied Biosystems, Foster City, CA, USA). Spearman correlations were computed for expression levels assessed by microarray and RT-PCR methods in a subset of genes (BCL2, NM_000633; CCND2, NM_001759; NCF2, NM_000433; PIK3R1, NM_181523; PRKCH, NM_006255) for whom correlations between gene expression and methylation levels were observed. Samples were analyzed in duplicate using predesigned probes (Hs00608023_ml, Hs00153380_m1, Hs01084940_m1, Hs00933163_ml and Hs00178933_ml) and calibrated to the ACTB housekeeping gene (endogenous control; ACTB: Hs99999903_m1). Relative quantification estimations were performed on an Applied Biosystems 7500 Real-Time PCR System (Applied Biosystems, Foster City, CA, USA).

2.4. Pathway Analysis. Analysis of potentially altered pathways was conducted using the knowledge base of the Ingenuity Pathway Analysis (IPA) system. The lists of 14466 differentially methylated and 862 differentially expressed probes produced from differential methylation and differential expression analysis, respectively, were submitted to IPA. Genes from each of these lists were classified according to pathways. IPA measured the likelihood that these genes participate in a particular pathway and calculated $P$ values using a right-tailed Fisher's exact test for each pathway. Overrepresented pathways were then obtained from differential methylation and expression analysis.

2.5. Statistical Analysis. Clinical data were expressed as mean $\pm \mathrm{SD}$. The effect of bariatric surgery in mothers was assessed using a within-subject paired $t$-test. Differences in anthropometric data between BMS and AMS siblings were tested using analysis of variance (general linear model, type III sum of squares) with adjustments for the effects of sex and puberty. In the absence of Tanner scores, we arbitrarily defined puberty as 12 years for girls and 14 for boys. Differences in severe obesity between BMS and AMS siblings were evaluated using BMI percentile and BMI $Z$-score and tested using Fisher's exact test. $P$ values for CRP were adjusted for the effects of sex, puberty and BMI percentile, an age- and sexcorrected adiposity measurement. Unadjusted $P$ values are also presented. Transformations were applied to nonnormally distributed variables (negative inverse transformed: CRP). Pairwise Pearson correlations between methylation, expression, and plasma CRP were computed for the IL-8 signaling pathway that was overrepresented in both differential methylation and expression analyses. Partial Pearson correlations were also computed for the IL-8 signaling pathway after adjustments for age and sex. Statistical significance was defined as $P \leq 0.05$. Statistical analyses were conducted using the SAS software version 9.2 (SAS Institute Inc.).

\section{Results}

3.1. Impact of Bariatric Surgery in Mothers. A total of 20 mothers $(41.0 \pm 5.3$ years; mean $\pm \mathrm{SD})$ who had undergone biliopancreatic diversion were recruited. Mean postoperative follow up was 12 years and 2 months at the time of the study. Bariatric surgery induced dramatic weight loss in mothers. On average, the women weighted $121.5 \pm 19.2 \mathrm{~kg}(\mathrm{BMI}=45.0 \pm$ $7.2)$ at the time of the surgery and $74.8 \pm 11.9 \mathrm{~kg}(\mathrm{BMI}=27.6 \pm$ 4.8 ) at the recruitment interview. The mean weight loss of $46.7 \pm 16.1 \mathrm{~kg}$ was associated with significant improvements in plasma lipids ( $P \leq 0.01$ for TG, HDL-C, LDL-C, total-C, and total-C/HDL-C ratio) and reductions in insulin resistance (HOMA-IR index; $P \leq 0.001$ ) and blood pressure (SBP and DBP; $P \leq 0.001)$ with a trend toward lower plasma glucose levels $(P=0.06)$.

\subsection{Inflammatory Marker in BMS versus AMS Offspring.} The 50 BMS and AMS offspring of 20 mothers were aged between 2 years and 8 months and 24 years and 11 months with similar sex distribution (40\% males in both BMS and AMS). BMS offspring were born 3 years and 5 months before and AMS offspring were born 3 years and 7 months after maternal bariatric surgery. BMS siblings were older (mean 
age 14.9 y versus 9.6 y) than AMS siblings (Table 1). Following adjustments for the effects of sex and puberty, AMS offspring showed a trend toward lower body fat percent $(P=0.07)$. There was a significantly lower prevalence of severe obesity in AMS using BMI percentile $(P=0.01)$ or BMI $Z$-score $(P=0.05)$ as indicators. AMS offspring had improved fasting insulin levels $(P=0.03)$, lower HOMA-IR index $(P=$ 0.03), and blood pressure. Plasma CRP levels were different in BMS versus AMS offspring (5.14 \pm 7.90 versus $3.58 \pm 11.09$; $P=0.03)$ even after taking into account the sex effect $(P=$ 0.03 ). These differences in plasma CRP levels were no longer significant after further adjustments for the effects of puberty, and BMI (BMI percentile).

3.3. Comparison of Pathways Identified by Differential Methylation and Expression Analyses. From the list of 5698 differentially methylated genes between BMS and AMS offspring, IPA revealed 160 pathways significantly overrepresented $(P<$ 0.05). Similar analysis conducted from the list of 862 differentially expressed genes between BMS and AMS offspring identified 68 overrepresented pathways. The list of the top 20 overrepresented inflammatory and immune pathways identified from differential methylation analysis along with corresponding results obtained from gene expression analysis is presented in Table 2. Among them, 5 pathways were found to be overrepresented in both methylation and expression: IL8 signaling, iCOS-iCOSL signaling in T-helper cells, role of NFAT in regulation of the immune response, B-cell receptor signaling, and glucocorticoid receptor signaling.

3.4. Correlation of Methylation and Expression Data for the Il-8 Signaling Pathway. Among the 5 pathways listed above, the IL- 8 pathway was the most significantly overrepresented from differential methylation analysis. It was thus selected for further analyses to highlight the potential link between gene methylation, expression, and inflammation. Among the 193 genes assigned to the IL- 8 signaling pathway by IPA, 70 genes represented by 214 methylation probes were found to be differentially methylated in BMS versus AMS (Supplementary Table 2). Corresponding expression data were available for 102 of these probes ( 46 genes). Among the 46 genes analyzed, correlations between DNA methylation and expression were significant for 17 genes (23 methylation probes; Table 3). Further analyses were then focused on these 17 genes to correlate gene methylation and expression with CRP levels. There were significant correlations between gene methylation and plasma CRP levels for 16 genes. Gene expression levels of 5 genes were found to correlate with plasma CRP levels (Table 3). All 5 genes with significant correlations between gene expression and plasma CRP levels (BCL2, CCND2, NCF2, PIK3R1, and PRKCH) also demonstrated correlations between methylation and expression and between gene methylation and plasma CRP levels (Table 3). Following adjustments for the effects of age and sex, similar results were obtained (Supplementary Table 3). All 17 genes initially showing correlation between gene methylation and expression levels demonstrated significant correlation after adjustments. In addition, the $\mathrm{RHOH}$ gene (methylation
TABLE 1: Offspring characteristics.

\begin{tabular}{|c|c|c|c|}
\hline & BMS & AMS & $P$-values ${ }^{1}$ \\
\hline$N$ (males) & $25(10)$ & $25(10)$ & \\
\hline Age (years) & $14.9 \pm 6.2$ & $9.6 \pm 5.3$ & 0.002 \\
\hline \multicolumn{4}{|l|}{ Anthropometric data } \\
\hline BMI percentile & $69.2 \pm 40.7$ & $65.7 \pm 32.6$ & 0.80 \\
\hline BMI $Z$-score & $1.84 \pm 2.08$ & $0.85 \pm 1.47$ & 0.22 \\
\hline Fat percent ${ }^{2}$ & $29.9 \pm 13.9$ & $21.4 \pm 10.3$ & 0.07 \\
\hline \multicolumn{4}{|l|}{ Severe obesity } \\
\hline BMI percentile $>98 \%(N)$ & 12 & 3 & 0.01 \\
\hline BMI $Z$-score $>3(N)$ & 7 & 1 & 0.05 \\
\hline \multicolumn{4}{|l|}{ Blood pressure } \\
\hline SBP (mm Hg) & $111.3 \pm 14.7$ & $97.4 \pm 14.6$ & 0.01 \\
\hline $\mathrm{DBP}(\mathrm{mm} \mathrm{Hg})$ & $64.6 \pm 9.8$ & $53.4 \pm 12.8$ & 0.002 \\
\hline \multicolumn{4}{|l|}{ Lipid profile ${ }^{3}$} \\
\hline Total-C (mmol/L) & $4.43 \pm 0.66$ & $4.21 \pm 0.58$ & 0.24 \\
\hline LDL-C (mmol/L) & $2.65 \pm 0.55$ & $2.53 \pm 0.58$ & 0.53 \\
\hline HDL-C (mmol/L) & $1.30 \pm 0.29$ & $1.30 \pm 0.25$ & 0.54 \\
\hline $\mathrm{TG}(\mathrm{mmol} / \mathrm{L})$ & $1.04 \pm 0.43$ & $0.82 \pm 0.37$ & 0.21 \\
\hline Total-C/HDL-C & $3.56 \pm 0.93$ & $3.37 \pm 0.85$ & 0.87 \\
\hline \multicolumn{4}{|l|}{ Glucose metabolism $^{3}$} \\
\hline Fasting glucose $(\mathrm{mmol} / \mathrm{L})$ & $4.92 \pm 0.43$ & $4.71 \pm 0.43$ & 0.22 \\
\hline Insulin $(\mu \mathrm{U} / \mathrm{mL})$ & $18.82 \pm 12.20$ & $11.27 \pm 7.36^{*}$ & 0.03 \\
\hline Homa-IR & $4.27 \pm 3.23$ & $2.43 \pm 1.72^{*}$ & 0.03 \\
\hline
\end{tabular}

Values are presented as mean \pm SD.

${ }^{1} P$-values adjusted for sex and puberty except for BMI percentile and BMI $Z$-score.

${ }^{2}$ Fat percent at 6 years or more (BMS, $N=23$; AMS, $N=17$ ).

${ }^{3}$ BMS, $N=25$; AMS, $N=21$.

Abbreviations: AMS: after maternal surgery; BMS: before maternal surgery; BMI: body mass index; SBP and DBP: systolic diastolic and systolic blood pressure; Total-C: total cholesterol; LDL-C: low-density lipoprotein cholesterol; HDL-C: high-density lipoprotein cholesterol; TG: triglycerides.

probe cg26163153) reached significance level while initially showing a trend toward significant correlation $(P=0.09)$. From the 16 genes correlated with CRP levels, 13 remained significant following adjustments. Only one (PIK3R1) of the five genes with significant correlations between gene methylation, expression, and CRP levels remained significant after adjustments, while trends toward significant correlation were found for BCL2, CCND2, and NCF2. Validation of gene expression microarray data for these 5 genes demonstrated significant correlations between microarray and RT-PCR results for 4 of the genes assessed (CCND2, $r=0.358, P=$ 0.02; NCF2, $r=0.574, P<0.0001 ; P I K 3 R 1, r=0.349$, $P=0.02$; PRKCH, $r=0.462, P=0.001)$, while BCL2 did not reach significance $(r=0.223, P=0.14)$.

\section{Discussion}

We demonstrated here for the first time that surgical treatment of severe maternal obesity results in sustained differences in the methylome and transcriptome of genes involved 
TABLE 2: Comparison of the top 25 overrepresented canonical immune/inflammatory pathways identified from differential methylation and expression analyses between BMS and AMS siblings.

\begin{tabular}{|c|c|c|c|c|}
\hline IPA canonical pathways & $P$ value & $\begin{array}{l}N \text { of diff. } \\
\text { meth. genes }\end{array}$ & $P$-value & $\begin{array}{c}N \text { of Diff. } \\
\text { Express. genes }\end{array}$ \\
\hline CD28 signaling in T-helper cells & $4.24 \times 10^{-7}$ & 54 & 0.40 & 5 \\
\hline Leukocyte extravasation signaling & $6.34 \times 10^{-7}$ & 78 & 0.25 & 13 \\
\hline Role of NFAT in cardiac hypertrophy & $6.98 \times 10^{-7}$ & 76 & NI & - \\
\hline T-Cell receptor signaling & $7.55 \times 10^{-7}$ & 47 & 0.07 & 7 \\
\hline Dendritic cell maturation & $8.22 \times 10^{-7}$ & 66 & 0.10 & 9 \\
\hline Clathrin-mediated endocytosis signaling & $1.24 \times 10^{-6}$ & 74 & 0.09 & 11 \\
\hline IL-8 signaling & $2.44 \times 10^{-6}$ & 70 & 0.03 & 12 \\
\hline iCOS-iCOSL signaling in T-helper cells & $5.35 \times 10^{-6}$ & 48 & 0.01 & 9 \\
\hline Virus entry via endocytic pathways & $5.48 \times 10^{-6}$ & 43 & NI & - \\
\hline$N F-\kappa B$ signaling & $6.08 \times 10^{-6}$ & 66 & 0.08 & 10 \\
\hline Role of NFAT in regulation of the immune response & $8.14 \times 10^{-6}$ & 69 & 0.02 & 12 \\
\hline PKC $\theta$ signaling in T lymphocytes & $9.18 \times 10^{-6}$ & 50 & 0.24 & 6 \\
\hline Macropinocytosis signaling & $2.5 \times 10^{-5}$ & 32 & NI & - \\
\hline CCR5 signaling in macrophages & $2.66 \times 10^{-5}$ & 34 & 0.42 & 3 \\
\hline CTLA4 Signaling in Cytotoxic T Lymphocytes & $2.71 \times 10^{-5}$ & 40 & NI & - \\
\hline B-Cell receptor signaling & $2.96 \times 10^{-5}$ & 58 & 0.008 & 12 \\
\hline fMLP signaling in neutrophils & $3.94 \times 10^{-5}$ & 47 & 0.05 & 8 \\
\hline RAR activation & $4.32 \times 10^{-5}$ & 66 & 0.32 & 8 \\
\hline IL-1 signaling & $6.55 \times 10^{-5}$ & 40 & 0.27 & 5 \\
\hline Glucocorticoid receptor signaling & $6.72 \times 10^{-5}$ & 92 & 0.005 & 19 \\
\hline
\end{tabular}

Pathways in bold are overrepresented in both gene methylation and expression data. Abbreviations: diff. express.: differentially expressed; diff. Meth.: differentially methylated; $N$ : number; NI: not identified.

in inflammatory pathways in AMS offspring compared to BMS siblings. The proportion of $34.7 \%$ of differentially methylated genes (1976 of 5698 genes) being identified by two probes or more strengthen, the validity of our results arguing for a regulation of methylation levels in offspring. Differential analysis of gene methylation and expression levels revealed important differences between the sibling groups. Pathway analysis from differentially methylated genes in BMS versus AMS revealed important regulation of inflammatory and immunity pathways: 29 inflammatory- or immunity-related pathways being identified among the top 50 overrepresented pathways (data not shown). Pathway analysis from differentially expressed genes between BMS and AMS revealed 5 overrepresented pathways among the top overrepresented pathways from differential methylation data (Table 2). Overrepresentation of genes from the IL-8 signaling pathway was identified from both methylation and expression data, and significant correlations were found between gene methylation and expression levels. Moreover, 5 genes from the IL-8 pathway also demonstrated correlation of gene methylation and expression levels with plasma CRP levels. Despite the fact that the impact of DNA methylation on gene expression seems to be site and location dependent, results obtained here for the IL-8 pathway generally comply to the known phenomenon that intragenic methylation (gene body, $5^{\prime}$ UTR and $3^{\prime}$ UTR) correlates with increased gene expression, while promoter methylation is associated with decreased expression [30, 31].
Gene expression profiling in obese patients who had undergone weight loss or bariatric surgery demonstrated a regulation of inflammation-related transcripts and changes in inflammatory marker levels $[32,33]$. In the present study, we extend these data by reporting that metabolic improvements in mothers following biliopancreatic diversion surgery are reflected in gene methylation and expression levels of immunologic and inflammatory genes in offspring, indeed reinforcing the involvement of epigenetic differences in immunity and inflammatory genes in the determination of the offspring phenotypes. A limited number of epigenetic studies have evaluated the impact of maternal nutrition and obesity on methylation of immune and inflammatory genes in offspring. Recently, human studies have revealed the implication of immune and inflammatory gene methylation in children with respect to adiposity. Methylation levels of the $R X R A$ gene in umbilical cord tissue DNA of healthy neonates were associated with maternal carbohydrate intake in early pregnancy and childhood adiposity at 9 years old [15]. Relton and collaborators reported associations between anthropometric indices (BMI, fat mass, lean mass, and height) in 9 years old children and methylation levels of selected immune and inflammatory genes in cord blood samples [34]. With a different design involving siblings born under different maternal obesity condition, our study supports the role of epigenetic factors in immunity and inflammatory genes in the determination of offspring phenotypes. 


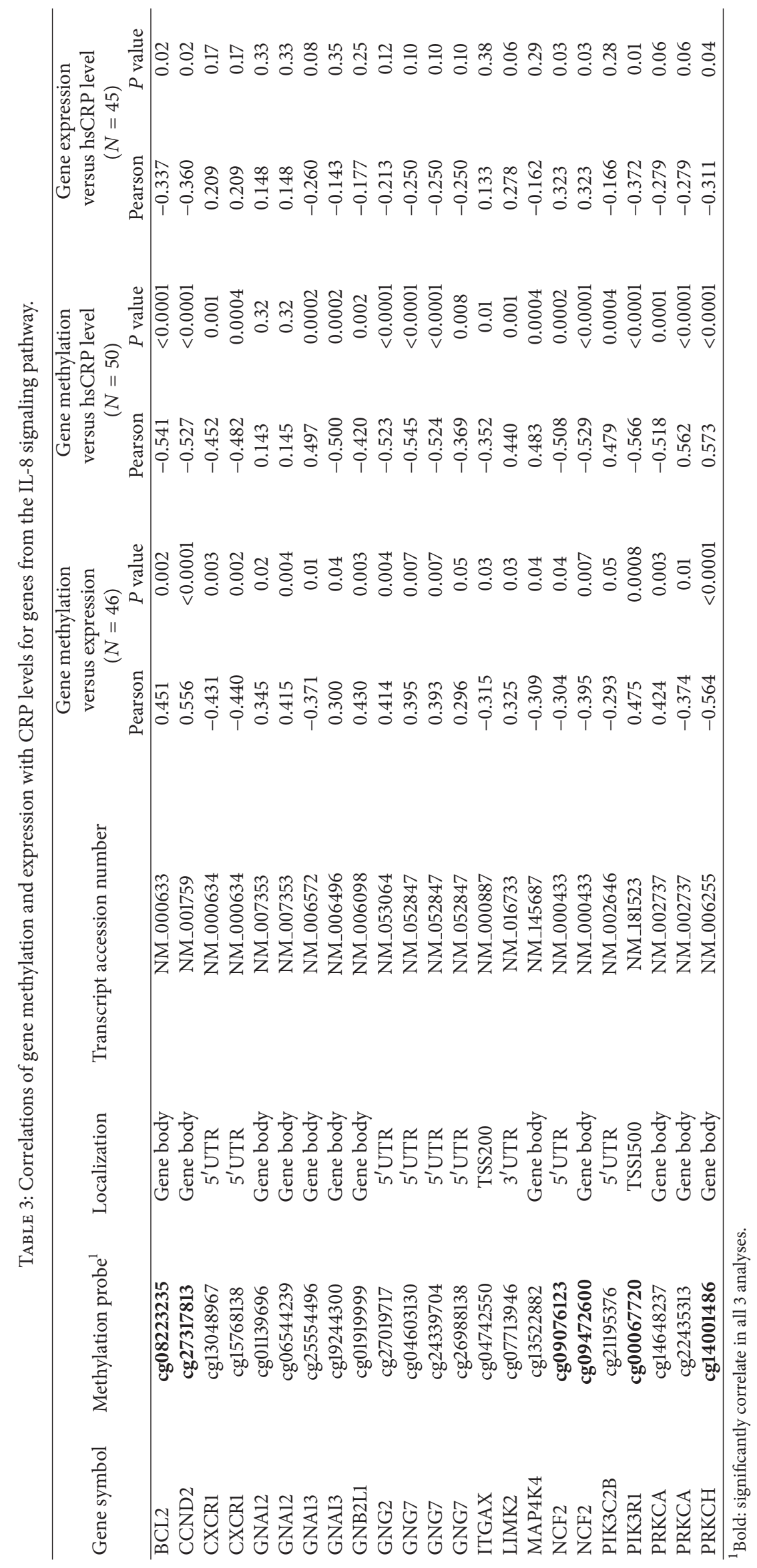


Transcriptomic data revealed that improvements seen in mothers following bariatric surgery are reflected in gene expression levels of immunologic and inflammatory genes in offspring. Animal studies have demonstrated a similar impact of maternal obesity and nutrition on immune and inflammatory genes in the offspring [35-37]. These results are consistent with those obtained from the present gene expression analysis in BMS offspring versus AMS siblings and may provide potential mechanisms for higher levels of systemic inflammation found in BMS offspring.

The present study has several limitations. We used DNA extracted from blood as it is more convenient and acceptable than biopsies of target tissues, especially in children. Although epigenetic signatures have been shown to differ between tissues [38], the overall impact is minor given the known similarities in methylation patterns [39]. Gene expression levels are also known to be tissue-or cell-type dependent but also correlated [40-42] thus justifying the use of blood in clinical studies. Differential gene expression analysis led to a limited number of differentially expressed genes (862) in comparison with the 5698 differentially methylated genes identified. This discrepancy may be attributable to differences in statistical algorithms, in corrections for multiple testing, or in the different cutoffs used; a cutoff fold change $\geq 1.2$ or $\leq 0.83$ was applied for gene expression analysis. Nonetheless, pathway analyses conducted from differentially methylated and expressed genes are concordant and demonstrate an overrepresentation of immune and inflammatory pathways. We did not stratify for sex in these gene methylation and expression analyses; several studies did not find sex-specific autosomal methylation patterns $[43,44]$. Owing to the before-after design of the study, we were unable to fully correct for the age difference between the sibling groups. This design and the young age of the AMS offspring (80\% being younger than 12 years old) do not allow discussing the long-term effects in children. Longitudinal studies are thus needed to further assess long-term effects of maternal bariatric surgery in the offspring. Age-related differential methylation has been reported but was shown to be siteand location dependent [45-47] and represented a very small proportion of $\mathrm{CpG}$ sites [47]. Furthermore, longitudinal studies in humans demonstrated relative stability of DNA methylation over time [39]. In regards to the general conclusion drawn from our results, various factors may be perceived as potential bias toward inflammatory effects. The potential impact of maternal weight regain on offspring is limited in the current study considering the sustained weight loss achieved with the biliopancreatic diversion with duodenal switch $[19,48,49]$. Analysis of maternal clinical files along with the lack of inflammation-related perioperative complications in biliopancreatic diversion with duodenal switches [48] argues against the potential impact of specific inflammatory condition in mothers. The current study was not designed to analyze methylation and expression changes induced in mothers following bariatric surgery. However, based on a recent meta-analysis demonstrating a reduction in low-grade inflammation associated with obesity following bariatric surgery [22], it would be tempting to speculate that these changes may be accompanied by similar changes in methylation and expression levels. Improvements in inflammatory profiles in mothers following bariatric surgery may be potentially responsible for the inflammatory effects seen in offspring through modification of the in utero environment and improved methylation profiles in AMS offspring.

\section{Conclusion}

Using analysis of gene methylation and expression data, we reveal that improvements seen in mothers following bariatric surgery reflect on the expression and methylation levels of genes involved in immune and inflammatory pathways in the offspring. These results argue for a beneficial effect of maternal weight loss before pregnancy and provide potential mechanisms of action for physiological improvements through regulation of methylation and expression of genes involved in inflammatory and immune pathways. Since inflammation is recognized as an independent risk factor for cardiovascular diseases (CVD) and is also associated with the development of the metabolic syndrome, insulin resistance, hypertension, and obesity $[50,51]$, it would be important to conduct longitudinal studies to examine the long-term effect of maternal bariatric surgery on offspring.

\section{Conflict of Interests}

The authors declare that there is no conflict of interests associated with this PAPER.

\section{Acknowledgments}

This study was supported by a Grant from the Canadian Institutes of Health Research (CIHR MOP-209380). The authors wish to thank all families who participated in the study for their excellent collaboration. The authors also want to recognize the collaboration of the members of the department of bariatric surgery, Laurent Biertho, Simon Biron, FrédéricSimon Hould, Stéfane Lebel, Odette Lescelleur, and Simon Marceau, for their involvement in the project. The authors express their gratitude to Suzy Laroche for help in sample and clinical information collection and Paule Marceau for subject recruitment, data management and project coordination. The authors would like to acknowledge the contribution of the McGill University and Génome Québec Innovation Centre for gene methylation and expression array processing and Mélissa Pelletier for inflammatory marker measurement. Frédéric Guénard is a recipient of a studentship award from the Heart and Stroke Foundation of Canada. André Tchernof holds a Research Chair in Bariatric and Metabolic Surgery. Katherine Cianflone is a Canada Research Chair in Adipose Tissue. Marie-Claude Vohl is a Canada Research Chair in Genomics Applied to Nutrition and Health.

\section{References}

[1] M. Z. Alfaradhi and S. E. Ozanne, "Developmental programming in response to maternal overnutrition," Frontiers in Genetics, vol. 2, p. 27, 2011. 
[2] S. B. Harrap, M. Stebbing, J. L. Hopper, H. N. Hoang, and G. G. Giles, "Familial patterns of covariation for cardiovascular risk factors in adults. The Victorian Family Heart Study," American Journal of Epidemiology, vol. 152, no. 8, pp. 704-715, 2000.

[3] K. L. Whitaker, M. J. Jarvis, R. J. Beeken, D. Boniface, and J. Wardle, "Comparing maternal and paternal intergenerational transmission of obesity risk in a large population-based sample," American Journal of Clinical Nutrition, vol. 91, no. 6, pp. 15601567, 2010.

[4] D. Dabelea, E. J. Mayer-Davis, A. P. Lamichhane et al., "Association of intrauterine exposure to maternal diabetes and obesity with type 2 diabetes in youth: the SEARCH case-control study," Diabetes Care, vol. 31, no. 7, pp. 1422-1426, 2008.

[5] T. A. Hillier, K. L. Pedula, M. M. Schmidt, J. A. Mullen, M.A. Charles, and D. J. Pettitt, "Childhood obesity and metabolic imprinting: the ongoing effects of maternal hyperglycemia," Diabetes Care, vol. 30, no. 9, pp. 2287-2292, 2007.

[6] H. Hochner, Y. Friedlander, R. Calderon-Margalit et al., "Associations of maternal prepregnancy body mass index and gestational weight gain with adult offspring cardiometabolic risk factors: the jerusalem perinatal family follow-up study," Circulation, vol. 125, no. 11, pp. 1381-1389, 2012.

[7] E. Villamor and S. Cnattingius, "Interpregnancy weight change and risk of adverse pregnancy outcomes: a population-based study," Lancet, vol. 368, no. 9542, pp. 1164-1170, 2006.

[8] J. E. Ramsay, W. R. Ferrell, L. Crawford, A. Michael Wallace, I. A. Greer, and N. Sattar, "Maternal obesity is associated with dysregulation of metabolic, vascular, and inflammatory pathways," Journal of Clinical Endocrinology and Metabolism, vol. 87, no. 9, pp. 4231-4237, 2002.

[9] T. Radaelli, J. Uvena-Celebrezze, J. Minium, L. Huston-Presley, P. Catalano, and S. Hauguel-de Mouzon, "Maternal interleukin6: marker of fetal growth and adiposity," Journal of the Society for Gynecologic Investigation, vol. 13, no. 1, pp. 53-57, 2006.

[10] M. J. Zhu, M. Du, P. W. Nathanielsz, and S. P. Ford, "Maternal obesity up-regulates inflammatory signaling pathways and enhances cytokine expression in the mid-gestation sheep placenta," Placenta, vol. 31, no. 5, pp. 387-391, 2010.

[11] M. J. Zhu, Y. Ma, N. M. Long, M. Du, and S. P. Ford, "Maternal obesity markedly increases placental fatty acid transporter expression and fetal blood triglycerides at midgestation in the ewe," American Journal of Physiology, vol. 299, no. 5, pp. R1224R1231, 2010.

[12] J. Dahlgren, C. Nilsson, E. Jennische et al., "Prenatal cytokine exposure results in obesity and gender-specific programming," American Journal of Physiology, vol. 281, no. 2, pp. E326-E334, 2001.

[13] G. C. Burdge, J. Slater-Jefferies, C. Torrens, E. S. Phillips, M. A. Hanson, and K. A. Lillycrop, "Dietary protein restriction of pregnant rats in the F0 generation induces altered methylation of hepatic gene promoters in the adult male offspring in the F1 and F2 generations," British Journal of Nutrition, vol. 97, no. 3, pp. 435-439, 2007.

[14] K. A. Lillycrop, E. S. Phillips, C. Torrens, M. A. Hanson, A. A. Jackson, and G. C. Burdge, "Feeding pregnant rats a proteinrestricted diet persistently alters the methylation of specific cytosines in the hepatic PPAR $\alpha$ promoter of the offspring," British Journal of Nutrition, vol. 100, no. 2, pp. 278-282, 2008.

[15] K. M. Godfrey, A. Sheppard, P. D. Gluckman et al., "Epigenetic gene promoter methylation at birth is associated with child's later adiposity," Diabetes, vol. 60, no. 5, pp. 1528-1534, 2011.
[16] K. Szarc vel Szic, M. N. Ndlovu, G. Haegeman, and W. Vanden Berghe, "Nature or nurture: let food be your epigenetic medicine in chronic inflammatory disorders," Biochemical Pharmacology, vol. 80, no. 12, pp. 1816-1832, 2010.

[17] A. E. Pontiroli, P. Pizzocri, M. C. Librenti et al., "Laparoscopic adjustable gastric banding for the treatment of morbid (grade 3) obesity and its metabolic complications: a three-year study," Journal of Clinical Endocrinology and Metabolism, vol. 87, no. 8, pp. 3555-3561, 2002.

[18] N. Scopinaro, G. M. Marinari, G. B. Camerini, F. S. Papadia, and G. F. Adami, "Specific effects of biliopancreatic diversion on the major components of metabolic syndrome: a long-term followup study," Diabetes Care, vol. 28, no. 10, pp. 2406-2411, 2005.

[19] P. Marceau, S. Biron, F.-S. Hould et al., "Duodenal switch: longterm results," Obesity Surgery, vol. 17, no. 11, pp. 1421-1430, 2007.

[20] A. E. Pontiroli, F. Folli, M. Paganelli et al., "Laparoscopic gastric banding prevents type 2 diabetes and arterial hypertension and induces their remission in morbid obesity: a 4-year casecontrolled study," Diabetes Care, vol. 28, no. 11, pp. 2703-2709, 2005.

[21] L. Sjöström, A.-K. Lindroos, M. Peltonen et al., "Lifestyle, diabetes, and cardiovascular risk factors 10 years after bariatric surgery," New England Journal of Medicine, vol. 351, no. 26, pp. 2683-2693, 2004.

[22] S. R. Rao, "Inflammatory markers and bariatric surgery: a metaanalysis," Inflammation Research, vol. 61, no. 8, pp. 789-807, 2012.

[23] J. G. Kral, S. Biron, S. Simard et al., "Large maternal weight loss from obesity surgery prevents transmission of obesity to children who were followed for 2 to 18 years," Pediatrics, vol. 118, no. 6, pp. e1644-e1649, 2006.

[24] J. Smith, K. Cianflone, S. Biron et al., "Effects of maternal surgical weight loss in mothers on intergenerational transmission of obesity," Journal of Clinical Endocrinology and Metabolism, vol. 94, no. 11, pp. 4275-4283, 2009.

[25] National Center for Health Statistics, "NHANES III clinical growth charts; children 2 to 20 years 5 th to 95 th percentile: boys' BMI for age and girls' BMI for age," National Center for Health Statistics, Hyattsville, Md, USA, 2007.

[26] National Center for Health Statistics, "NHANES III Zscore data files: BMI for age and sex 2 to 20 years," National Center for Health Statistics, Hyattsville, Md, USA, 2007.

[27] M.-C. Vohl, R. Sladek, J. Robitaille et al., "A survey of genes differentially expressed in subcutaneous and visceral adipose tissue in men," Obesity Research, vol. 12, no. 8, pp. 1217-1222, 2004.

[28] M. Blazejczyk, M. Miron, and R. Nadon, "FlexArray: a statistical data analysis software for gene expression microarrays," Genome Quebec, Montreal, Canada, 2007.

[29] V. G. Tusher, R. Tibshirani, and G. Chu, "Significance analysis of microarrays applied to the ionizing radiation response," Proceedings of the National Academy of Sciences of the United States of America, vol. 98, no. 9, pp. 5116-5121, 2001.

[30] P. A. Jones, "Functions of DNA methylation: islands, start sites, gene bodies and beyond," Nature Reviews Genetics, vol. 13, no. 7, pp. 484-492, 2012.

[31] T. A. Rauch, X. Wu, X. Zhong, A. D. Riggs, and G. P. Pfeifer, "A human B cell methylome at 100-base pair resolution," Proceedings of the National Academy of Sciences of the United States of America, vol. 106, no. 3, pp. 671-678, 2009. 
[32] K. Clément, N. Viguerie, C. Poitou et al., "Weight loss regulates inflammation-related genes in white adipose tissue of obese subjects," FASEB Journal, vol. 18, no. 14, pp. 1657-1669, 2004.

[33] A. R. Moschen, C. Molnar, S. Geiger et al., "Anti-inflammatory effects of excessive weight loss: potent suppression of adipose interleukin 6 and tumour necrosis factor a expression," Gut, vol. 59, no. 9, pp. 1259-1264, 2010.

[34] C. L. Relton, A. Groom, B. St. Pourcain et al., "DNA methylation patterns in cord blood DNA and body size in childhood," PLoS ONE, vol. 7, no. 3, Article ID e31821, 2012.

[35] K. Shankar, P. Kang, A. Harrell et al., "Maternal overweight programs insulin and adiponectin signaling in the offspring," Endocrinology, vol. 151, no. 6, pp. 2577-2589, 2010.

[36] X. Yan, Y. Huang, H. Wang et al., "Maternal obesity induces sustained inflammation in both fetal and offspring large intestine of sheep," Inflammatory Bowel Diseases, vol. 17, no. 7, pp. 15131522, 2011.

[37] K. Shankar, Y. Zhong, P. Kang et al., "Maternal obesity promotes a proinflammatory signature in rat uterus and blastocyst," Endocrinology, vol. 152, no. 11, pp. 4158-4170, 2011.

[38] H.-M. Byun, K. D. Siegmund, F. Pan et al., "Epigenetic profiling of somatic tissues from human autopsy specimens identifies tissue- and individual-specific DNA methylation patterns," Human Molecular Genetics, vol. 18, no. 24, pp. 4808-4817, 2009.

[39] R. P. Talens, D. I. Boomsma, E. W. Tobi et al., "Variation, patterns, and temporal stability of DNA methylation: considerations for epigenetic epidemiology," FASEB Journal, vol. 24, no. 9, pp. 3135-3144, 2010.

[40] H. R. Brattbakk, I. Arbo, and S. Aagaard, "Balanced caloric macronutrient composition downregulates immunological gene expression in human blood cells-adipose tissue diverges," Omics, vol. 17, no. 1, pp. 41-52, 2011.

[41] C.-C. Liew, J. Ma, H.-C. Tang, R. Zheng, and A. A. Dempsey, "The peripheral blood transcriptome dynamically reflects system wide biology: a potential diagnostic tool," Journal of Laboratory and Clinical Medicine, vol. 147, no. 3, pp. 126-132, 2006.

[42] I. Rudkowska, C. Raymond, A. Ponton et al., "Validation of the use of peripheral blood mononuclear cells as surrogate model for skeletal muscle tissue in nutrigenomic studies," Omics, vol. 15, no. 1-2, pp. 1-7, 2011.

[43] J. T. Bell, A. A. Pai, J. K. Pickrell et al., "DNA methylation patterns associate with genetic and gene expression variation in HapMap cell lines," Genome Biology, vol. 12, no. 1, article R10, 2011.

[44] F. Eckhardt, J. Lewin, R. Cortese et al., "DNA methylation profiling of human chromosomes 6, 20 and 22," Nature Genetics, vol. 38, no. 12, pp. 1378-1385, 2006.

[45] B. C. Christensen, E. A. Houseman, C. J. Marsit et al., "Aging and environmental exposures alter tissue-specific DNA methylation dependent upon CPG island context," PLoS Genetics, vol. 5, no. 8, Article ID e1000602, 2009.

[46] M. F. Fraga, R. Agrelo, and M. Esteller, "Cross-talk between aging and cancer: the epigenetic language," Annals of the New York Academy of Sciences, vol. 1100, pp. 60-74, 2007.

[47] V. K. Rakyan, T. A. Down, S. Maslau et al., "Human agingassociated DNA hypermethylation occurs preferentially at bivalent chromatin domains," Genome Research, vol. 20, no. 4, pp. 434-439, 2010.

[48] L. Biertho, S. Lebel, S. Marceau et al., "Perioperative complications in a consecutive series of 1000 duodenal switches," Surgery for Obesity and Related Diseases, vol. 9, no. 1, pp. 63-68, 2011.
[49] H. Buchwald, Y. Avidor, E. Braunwald et al., "Bariatric surgery: a systematic review and meta-analysis," Journal of the American Medical Association, vol. 292, no. 14, pp. 1724-1737, 2004.

[50] S. M. Haffner, "The metabolic syndrome: inflammation, diabetes mellitus, and cardiovascular disease," American Journal of Cardiology, vol. 97, no. 2, pp. 3A-11A, 2006.

[51] P. M. Ridker, N. Rifai, L. Rose, J. E. Buring, and N. R. Cook, "Comparison of C-reactive protein and low-density lipoprotein cholesterol levels in the prediction of first cardiovascular events," New England Journal of Medicine, vol. 347, no. 20, pp. 1557-1565, 2002. 


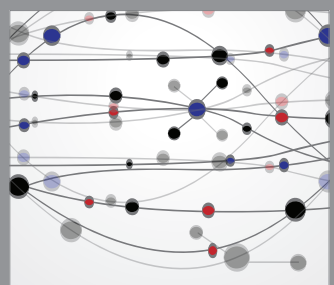

The Scientific World Journal
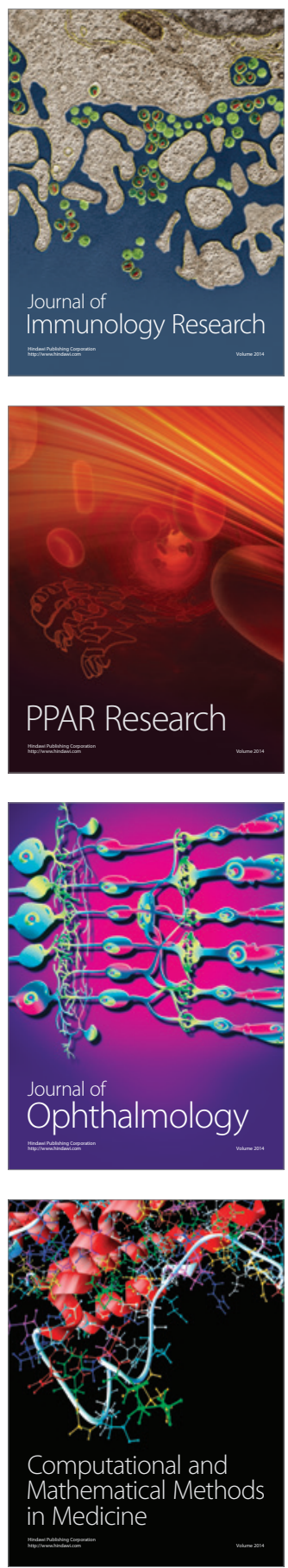

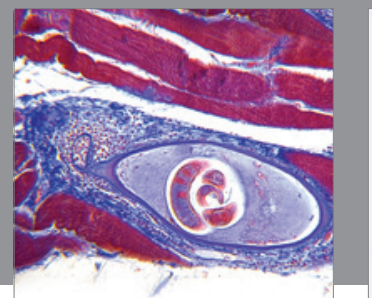

Gastroenterology

Research and Practice
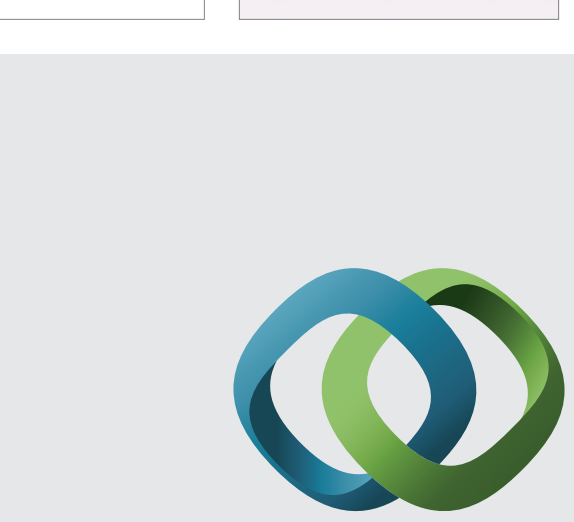

\section{Hindawi}

Submit your manuscripts at

http://www.hindawi.com
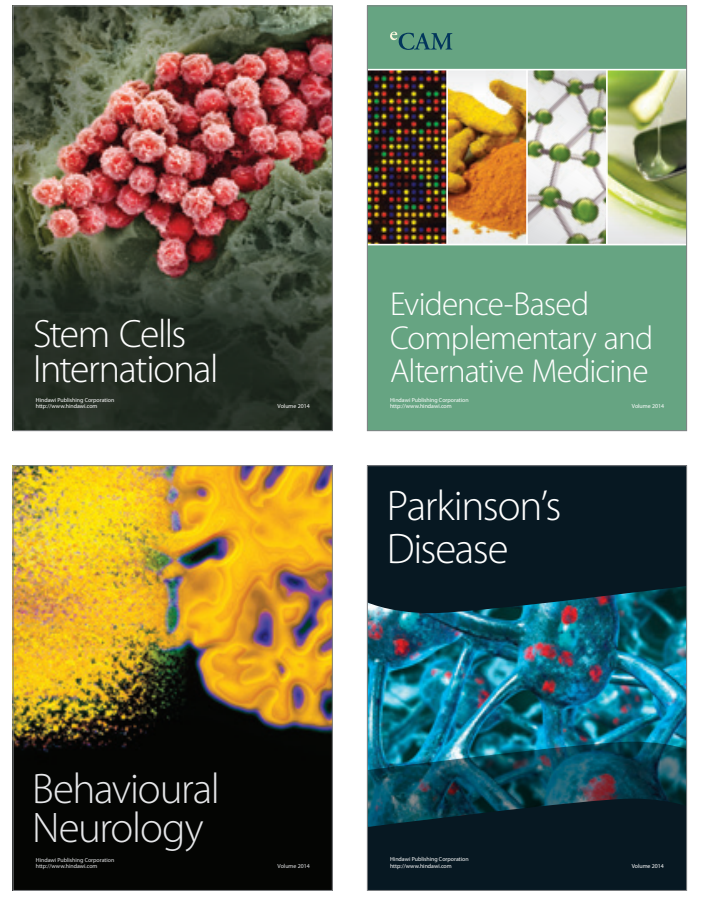
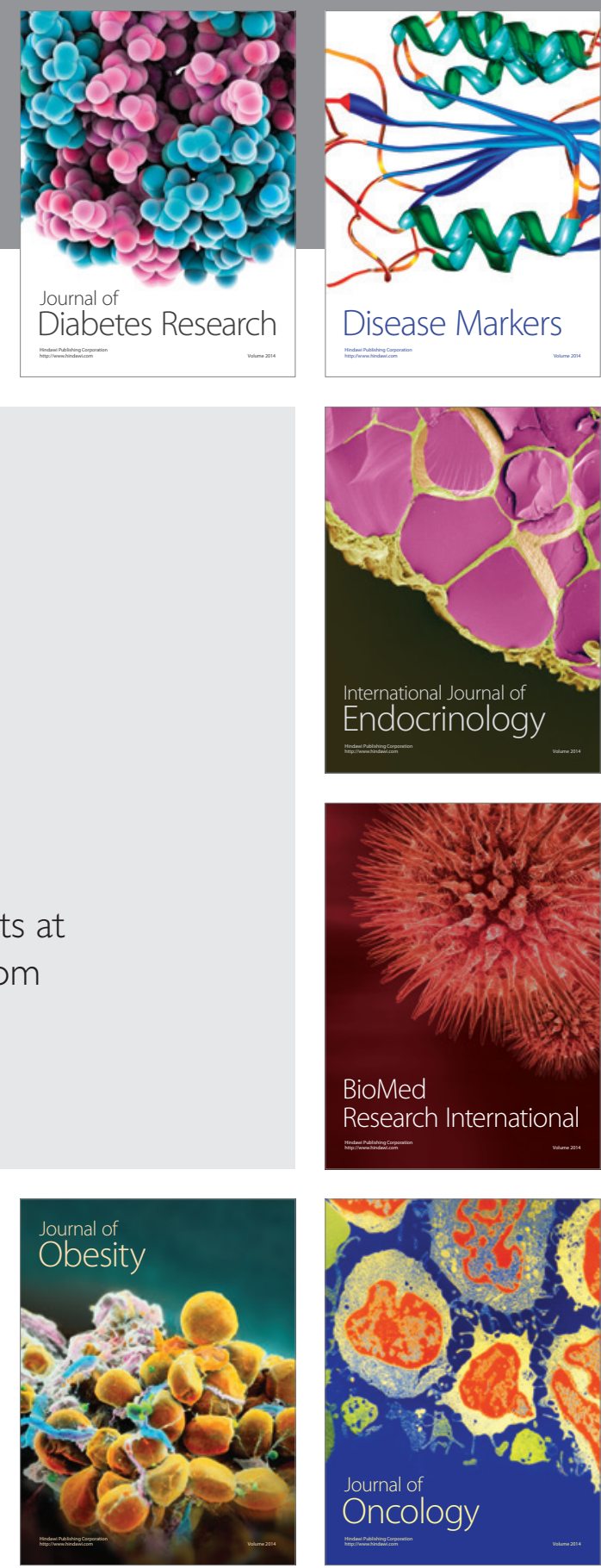

Disease Markers
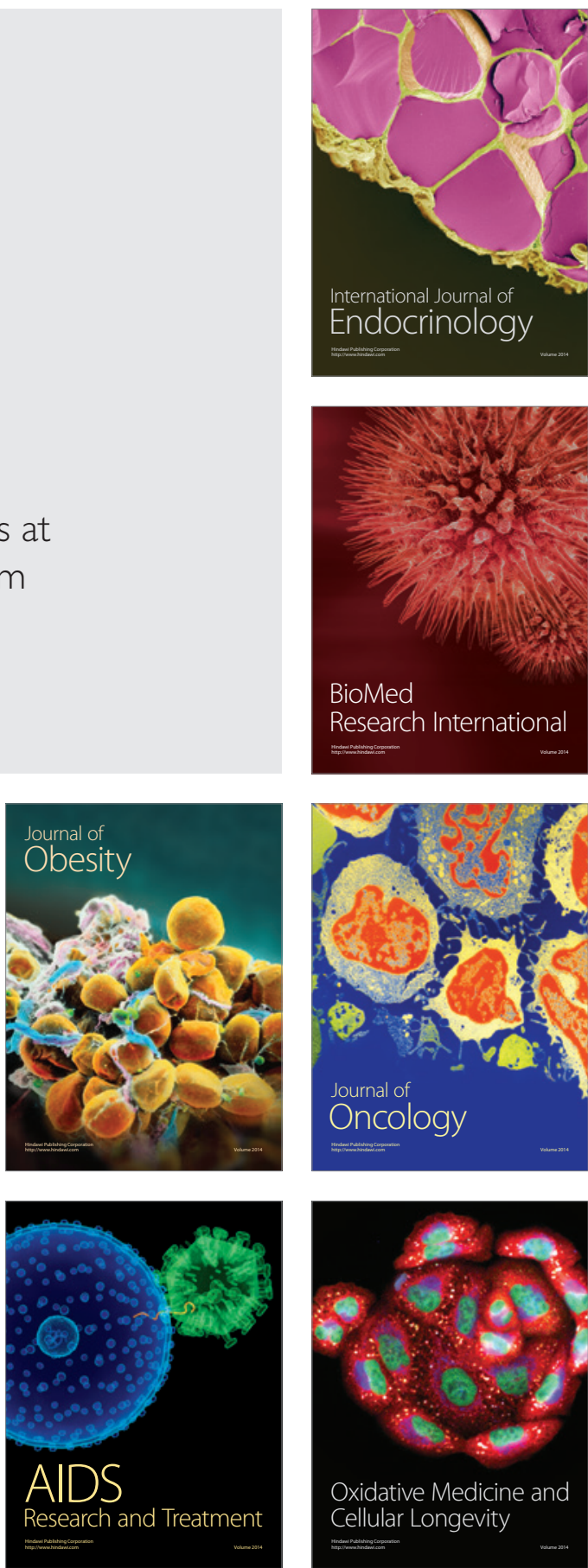